\title{
Double closed loop controller of wheeled mobile robot for trajectory tracking based on back-stepping and Lyapunov method
}

\author{
Limin Du \\ College of Electronic Information Engineering, Changchun University, Changchun, China \\ email: zkdlm911@163.com
}

Keywords: Wheeled mobile robot, Trajectory tracking, Back-stepping, Lyapunov, Kinematics model, Dynamics model

Abstract: A new trajectory tracking controller for a wheeled mobile robot based on backstepping and Lyapunov approach is presented. The proposed controller is developed based on the kinematic model and dynamics model of wheeled mobile robot. Firstly, the kinematic controller is designed by using back-stepping control method. Secondly, according to the dynamic model of multi input static coupling, the virtual control is used to decouple the dynamic model, and then the dynamic controller is designed by using the Lyapunov method. The output of the kinematic controller is used as the input reference of the dynamics controller. The simulation results show that the designed control scheme has a better dynamic performance and steady state performance, and has a certain practical value in tracking the trajectory of wheeled mobile robots system.

\section{Introduction}

Wheeled mobile robot has the advantage of light deadweight, big carrying, simple structure, relative convenience to drive and control, flexible mobility, high work efficiency, and is widely applied in industry, agriculture, anti-terrorism explosion-proof, family and space exploration and other fields. Robot has strong coupling, time-varying and nonlinear dynamic characteristics, so control is very complicated. In recent years, the motion control of wheeled mobile robot has been the research hotspot in the field of control. Some scholars have used nonlinear system control method in the control of wheeled mobile robot, such as the sliding mode variable structure control[1- 2], adaptive control[3-4] and back-stepping control[5-6]. At the same time, the different path tracking control strategies are also proposed in [7]. Among them, back-stepping control design method to deal with the problem of nonlinear system control has better control effect. Based on the back-stepping design method, reference [5-6] et al. solve the trajectory tracking problem of wheeled mobile robot, but only for the kinematic model , but for the actual value of the dynamic model is rarely considered.

In this paper, a double closed loop controller is designed based on the kinematics model and dynamics model of wheeled robot. Firstly, the back-stepping control law is designed based on the kinematics model, and the output of the back-stepping control law is used as the input reference of the inner loop dynamics model. Secondly, the dynamic model is decoupled according to the virtual control transformation, and then the control law is designed for the two first order differential equation of after decoupling by Lyapunov method.Finally, the simulation is carried out in 
matlab/simulink, and the results show that the control method designed in this paper has good tracking performance.

\section{System Controller Design}

The independent dual rear wheel differential drive mobile robot controls the speed and direction of the robot by different speeds of the two rear wheels. Two models are used, one is kinematics model, which is used to solve the problem of speed and position control, and the other is dynamic model, which is used to solve the control problem between speed and input force.

\subsection{Kinematic Control Law Design}

Assuming the dual rear wheel independent drive rigid mobile robot moving in the plane, and assuming that the absolute coordinates of the OXY fixed in the plane, then dual rear wheel drive mobile robot is shown in figure 1 .

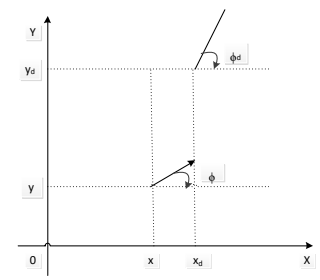

Figure 1 Position coordinate of mobile robot.

The state of the robot is represented by the position and direction angle $\phi$ of midpoint $\mathrm{M}$ in coordinate system. $\phi$ is the angle between the forward direction and the $\mathrm{X}$ axis, then the kinematics model of the robot is as follows:

$$
\left\{\begin{array}{l}
\dot{x}=v \cos \phi \\
\dot{y}=v \sin \phi \\
\dot{\phi}=\omega
\end{array}\right.
$$

where, $v$ and $\omega$ are respectively the robot's linear speed and angular speed.

First step: The virtual input $\gamma$ is introduced, according to the Eq. (1), has

$$
\left\{\begin{array}{l}
\cdot \\
x=v \cos \gamma \\
y=v \sin \gamma
\end{array}\right.
$$

$\left(x_{d}, y_{d}\right)$ is position setting value, $\left(x_{e}, y_{e}\right)$ is position tracking error, where, $x_{e}$ is equal to $x_{d}-x, y_{e}$ is equal to $y_{d}-y$, take the Lyapunov function as follows:

$$
V_{1}=\frac{1}{2} x_{e}^{2}+\frac{1}{2} y_{e}^{2}
$$

By Eq.(2), has 


$$
\dot{V_{1}}=x_{e} \dot{x}_{e}+y_{e} \dot{y}_{e}=x_{e}\left(\dot{x}_{d}-v \cos \gamma\right)+y_{e}\left(\dot{y}_{d}-v \sin \gamma\right)
$$

Make $x_{d}+k_{1} x_{e}$ equal to $m_{1}, y_{d}+k_{2} y_{e}$ equal to $m_{2}$, the linear velocity control law is designed as follows:

$$
\begin{aligned}
& v=\sqrt{m_{1}^{2}+m_{2}^{2}} \\
& \gamma=\arctan \frac{m_{2}}{m_{1}}=\arctan \frac{y_{d}+k_{2} y_{e}}{x_{d}+k_{1} x_{e}}
\end{aligned}
$$

then,

$$
\dot{V}_{1}=-k_{1} x_{e}^{2}-k_{2} y_{e}^{2}<0
$$

Second step: Make $e$ equal to $\gamma-\phi$, define the Lyapunov function as

$$
V_{2}=V_{1}+\frac{1}{2} e^{2}
$$

then,

$$
\dot{V}_{2}=\dot{V}_{1}+e \dot{e}=-k_{1} x_{e}^{2}-k_{2} y_{e}^{2}+e(\dot{\gamma}-\omega)
$$

The angular velocity control law is designed as follows:

$$
\omega=\gamma+k_{3} e
$$

then,

$$
\dot{V}_{2}=-k_{1} x_{e}^{2}-k_{2} y_{e}^{2}-k_{3} e^{2}<0
$$

According to the Lyapunov stability theory, the system (1) is asymptotically stable under the control law Eq.(5) and Eq.(9).

\subsection{Dynamic Control Law Design}

According to the principle of moment balance, for car body, has

$$
I_{m} \omega=F_{r} d-F_{l} d
$$

where, $I_{m}$ is the turn inertia around the center of gravity of the robot, $F_{F}$ and $F_{I}$ are respectively the driving forces of the left wheel and right wheel, $d$ is the distance between the center of gravity of the robot and the left wheel and right wheel.

According to Newton's law, has

$$
m_{c} \dot{v}=F_{r}+F_{1}
$$

where, $m_{c}$ is the car body weight. 
According to the principle of moment balance, for the left wheel and right wheel, respectively have

$$
\left\{\begin{array}{l}
I_{n} \omega_{l}+c \omega_{1}=k \mu_{1}-R F_{l} \\
I_{n} \omega_{r}+c \omega_{r}=k \mu_{r}-R F_{r}
\end{array}\right.
$$

where, $I_{n}$ is the turn inertia of wheel, $\omega_{1}$ and $\omega_{r}$ are rotation angular velocity of wheels, c is viscous friction coefficient, $\mathrm{k}$ is gain, $\mu_{l}$ and $\mu_{r}$ are control inputs. $\mathrm{R}$ is wheel radius.

By Eq.(13), has

$$
I_{n} R\left(\dot{\omega}_{l}+\dot{\omega}_{r}\right)+c R\left(\omega_{1}+\omega_{r}\right)=k R\left(\mu_{1}+\mu_{r}\right)-R^{2}\left(F_{l}+F_{r}\right)
$$

According to the principle of mobile robot, has

$$
\begin{aligned}
& v=\frac{v_{r}+v_{1}}{2} \\
& d \omega=\frac{v_{r}-v_{1}}{2}
\end{aligned}
$$

and

$$
\begin{aligned}
& v_{r}=R \omega_{r} \\
& v_{1}=R \omega_{1}
\end{aligned}
$$

By Eq. (12), (14), (15) and (16), has

$$
\dot{v}=-\frac{2 c}{m_{c} R^{2}+2 I_{n}} v+\frac{k R}{m_{c} R^{2}+2 I_{n}}\left(\mu_{r}+\mu_{1}\right)
$$

By Eq. (13), has

$$
I_{n} R\left(\dot{\omega}_{1}-\dot{\omega}_{r}\right)+c R\left(\omega_{1}-\omega_{r}\right)=k R\left(\mu_{1}-\mu_{r}\right)-R^{2}\left(F_{1}-F_{r}\right)
$$

By Eq.(11), (15), (16) and (18), has

$$
\dot{\omega}=-\frac{2 c d^{2}}{I_{m} R^{2}+2 I_{n} d^{2}} \omega+\frac{k R d}{I_{m} R^{2}+2 I_{n} d^{2}}\left(\mu_{r}-\mu_{1}\right)
$$

The dynamic model of wheeled mobile robot is composed of formula (17) and formula (19), has

$$
\left\{\begin{array}{l}
\dot{v}=a_{1} v+b_{1}\left(\mu_{r}+\mu_{1}\right) \\
\dot{\omega}=a_{2} \omega+b_{2}\left(\mu_{r}-\mu_{1}\right)
\end{array}\right.
$$

where, $a_{1}=-\frac{2 c}{\left(m_{c} R^{2}+2 I_{n}\right)}, a_{2}=-\frac{2 c d^{2}}{I_{m} R^{2}+2 I_{n} d^{2}}, b_{1}=\frac{k R}{m_{c} R^{2}+2 I_{n}}, b_{2}=\frac{k R d}{I_{m} R^{2}+2 I_{n} d^{2}}$. 
It can be seen that the dynamic model of wheeled mobile robot has coupling part. Now, we introduce the virtual control quantity $u_{1}$ and $u_{2}$, and make $u_{r}$ equal to $u_{1}-u_{2}, u_{1}$ equal to $u_{2}$, then Eq. (20) becomes

$$
\begin{gathered}
\dot{v}=a_{1} v+b_{1} u_{1} \\
\dot{\omega}=a_{2} \omega+b_{2} u_{1}-2 b_{2} u_{2}
\end{gathered}
$$

$v_{d}$ and $\omega_{d}$ are the output of kinematic controller. Linear velocity error is $v_{e}$. Angular velocity error is $\omega_{e} \cdot v_{e}$ is equal to $v_{d}-v$. $\omega_{e}$ is equal to $\omega_{d}-\omega$.

For Eq. (21), define the Lyapunov function as

$$
V_{3}=\frac{1}{2} v_{e}^{2}
$$

then,

$$
\dot{V}_{3}=v_{e} \dot{v}_{e}=v_{e}\left(\dot{v}_{d}-a_{1} v-b_{1} u_{1}\right)
$$

The control law $u_{1}$ is designed as follows:

$$
u_{1}=\frac{1}{b_{1}}\left(k_{4} v_{e}+\dot{v}_{d}-a_{1} v\right)
$$

then,

$$
\dot{V}_{3}=-k_{4} v_{e}^{2}<0
$$

For Eq. (22), define the Lyapunov function as

$$
V_{4}=\frac{1}{2} \omega_{e}^{2}
$$

then,

$$
\dot{V}_{4}=\omega_{e} \dot{\omega}_{e}=\omega_{e}\left(\dot{\omega}_{d}-a_{2} \omega-b_{2} u_{1}+2 b_{2} u_{2}\right)
$$

The control law $u_{2}$ is designed as follows:

$$
u_{2}=\frac{1}{2 b_{2}}\left(-\dot{\omega}_{d}+a_{2} \omega+b_{2} u_{1}-k_{5} \omega_{e}\right)
$$

then,

$$
\dot{V}_{4}=-k_{5} \omega_{e}^{2}<0
$$

According to the Lyapunov stability theory, the system (21) and (22) are asymptotically stable under the control law (25) and (29). 


\section{Experimental results and analysis}

$x_{d}=\sin (t), y_{d}=\cos (t)$. The initial position of the controlled object is [0.5 0.50$]$. The parameters of kinematics controller and dynamics Controller are selected for $k_{1}=9, k_{2}=9, k_{3}=48, k_{4}=9, k_{5}=9$.The amplitudes of the control input $u_{r}$ and $u_{1}$ are limited to 9. Simulation has been carried out in MATLAB/Simulink. The simulation results are shown in Fig.(2) to (8).

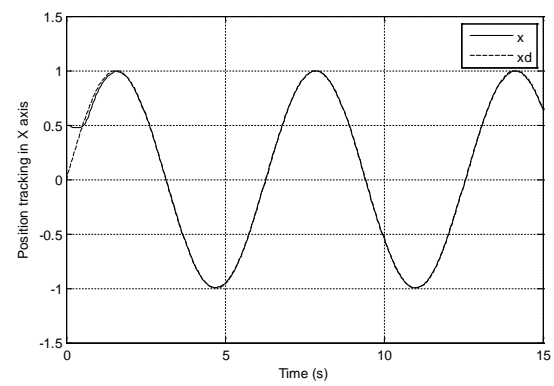

Figure 2 Position tracking in $\mathrm{X}$ axis

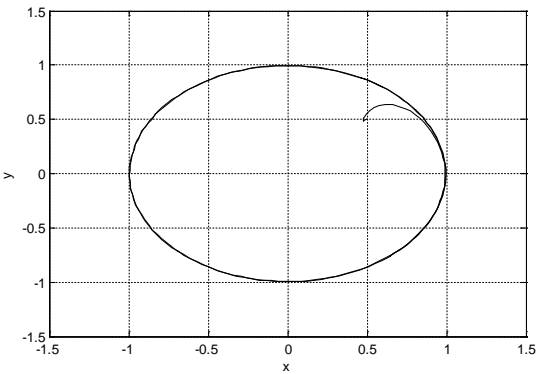

Figure 4 Position tracking curve

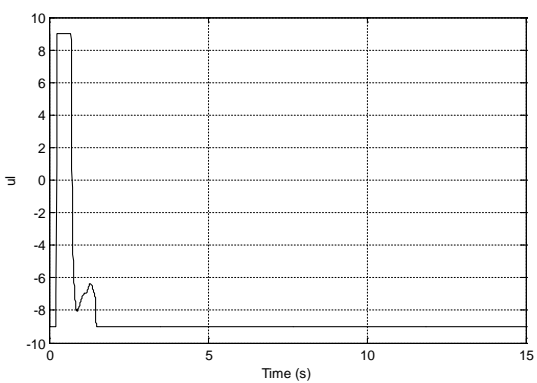

Figure 6 Control input ul

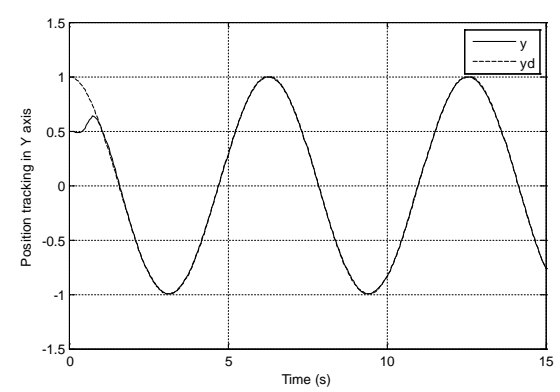

Figure 3 Position tracking in $\mathrm{Y}$ axis

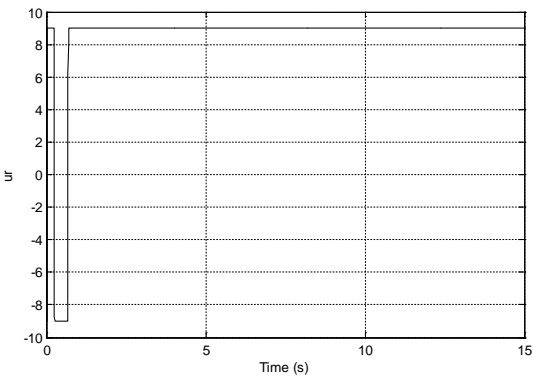

Figure 5 Control input ur

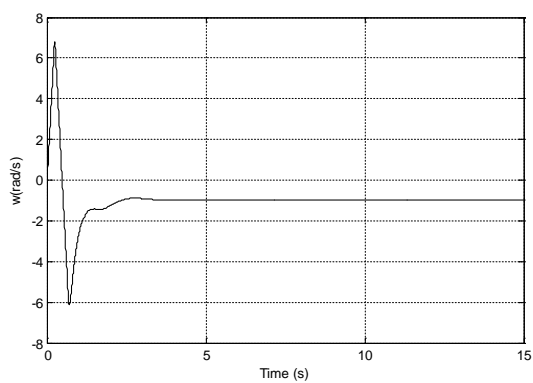

Figure 7 Angular speed curve

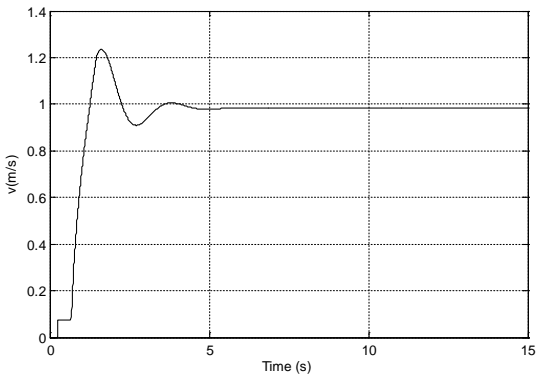

Figure 8 Linear speed curve 
Fig.(2), (3) and (4) are the position tracking curve, it can be seen from the charts, despite the initial error, but under the action of control law, trajectory tracking is still achieved, after 4 seconds, the tracking error converges to a small interval. Fig. (5) and Fig.(6) are the control signals which are limited. Fig. (7) and Fig.(8) are the angular velocity and linear velocity curves.

\section{Conclusions}

Aiming at the trajectory tracking problem of wheeled mobile robot, a double closed loop control based on kinematics model and dynamics model is proposed in this paper. The outer loop is a kinematic controller, and the back-stepping control method is adopted. The inner loop is a dynamic control, and the Lyapunov method is adopted. The simulation results show that the designed control law can ensure the accuracy and stability of trajectory tracking.

\section{Acknowledgment}

The work is financially supported by the plan project of Education Department of Jilin Province of China (2015LY502L13)

\section{References}

[1] YANG Min,MEI Jinsong and LIAO Licheng.(2015) Design of Backstepping-based Sliding-mode Trajectory Tracking Controller for Nonholonomic Mobile Robot. Machine Building and Automation ,152-154,196.

[2] ZHANG Y L,CHUNG J H,VELINSKY S A.(2003) Variable structure control of a differentially steered wheeled mobile robot. Journal of Intelligent and Robotic Systems, 301-314.

[3] PANG Hai-long,MA Bao-li.(2014) Adaptive unified controller of arbitrary trajectory tracking for wheeled mobile robots with unknown parameters. Control Theory \& Applications,285-292.

[4] Veer Alakshendra; Shital S. Chiddarwar.(2016) A robust adaptive control of mecanum wheel mobile robot: simulation and experimental validation, 2016 IEEE/RSJ International Conference on Intelligent Robots and Systems (IROS),5606-5611.

[5] Walied M. E. Mahgoub; Ibrahim M. H. Sanhoury (2017) Back stepping tracking controller for wheeled mobile robot. International Conference on Communication, Control, Computing and Electronics Engineering (ICCCCEE), 1-5.

[6] WU Wei-Guo,CHEN Hui-Tang and WANG Yue-Juan.(2001) Global trajectory tracking control of mobile robots.ACTA AUTOMATICA SINICA,326-331.

[7] Zheng Z W,Huo W and Zhu B.(2012)Global path-following control for nonholonomic mobile robots. Control Theory \& Applications, 741-746. 